\title{
OBSERVACIONES SOBRE LAS AVENULA DEL GRUPO MARGINATA EN ANDALUCÍA
}

\author{
Carlos ROMERO ZARCO
}

RESUMEN. Observaciones sobre las Avenula del grupo marginata en Andalucía. En este artículo se defiende un concepto estricto del género para las avenas perennes (Poaceae). Se proponen nuevas combinaciones nomenclaturales para varios táxones incluidos en la especie antiguamente llamada Avena sulcata Gay ex Boiss., actualmente Avenula marginata (Love) J. Holub sensu lato y se aportan datos sobre sinonimia, distribución y variabilidad de estos táxones con especial referencia a Andalucía (España).

Palabras clave. Taxonomía, nomenclatura, corología, Avenula, Helictotrichon, Península Ibérica, Andalucía.

SUMMARY. Observations on Avenula marginata group in Andalousia. This paper supports a narrow concept of genus in relation to perennial oats (Poaceae). New nomenclatural combinations are proposed for several taxa included in the formerly named Avena sulcata Gay ex Boiss, actually Avenula marginata (Love) J. Holub sensu lato. Remarks on synonymy, distribution and variability of these taxa are given with special references to Andalousia (Spain).

Key words. Taxonomy, nomenclature, chorology, Avenula, Helictotrichon, Iberian Peninsula, Andalousia.

En la Península Ibérica y regiones adyacentes del Mediterráneo, existen varios complejos poliploides de gramíneas perennes que plantean interesantes y difíciles problemas taxonómicos cuyas complicaciones nomenclaturales y florísticas son numerosas.

El objetivo de este trabajo es poner al día el tratamiento taxonómico y la distribución del grupo de táxones más estrechamente relacionados con Avenula marginata (Lowe) J. Holub con especial referencia al territorio andaluz. Los datos concretos sobre los caracteres diagnósticos, anatómicos y cariológicos, así como la iconografía y testimonios de herbarios pueden consultarse en un trabajo anterior (Rome- ro Zarco, 1984a) y en otras referencias que se indican en el texto.

\section{Sobre la delimitación del género Avenula.}

En su excelente monografía sobre las avenas perennes Del Mediterráneo occidental, Röser (1989) vuelve a considerar a este grupo dentro de un único género, Helictotrichon Bess. ex Schultes \& Schultes fil. Este criterio ha sido admitido hasta el segundo tercio del presente siglo por varios autores (Henrard, 1940; Hitchcock, 1950; Holub, 1958, Paunero, 1959, Baum, 1968, y Bor, 1968), y reaparece en las obras sintéticas de Clayton \& Renvoize (1986) 
y de Watson \& Dallwitz (1992).

Aunque la separación de las avenas perennes en dos géneros tiene su precedente en los estudio anatómicos de Duval-Jouve (1863) y de Potztal (1951), su formulación concreta corresponde a Holub (1962) y a Gervais (1968, 1973), quienes en base a criterios morfológicos y anatómicos separan el género Avenula (Dumort.) Dumort. (= Avenochloa J. Holub) del género Helictotrichon Bess. ex Schultes \& Schultes fil.

Las dos alternativas básicas existentes en la clasificación de las avenas perennes y sus implicaciones respecto a otros géneros de la tribu Aveneae (Avena, Arrhenatherum, Pseudarrhenatherum, Amphibromus y Trisetum), plantean numerosas cuestiones sistemáticas y nomenclaturales cuya discusión ha llenado brillantes y extensas introducciones de varios artículos, entre los cuales me permito recomendar la lectura de Sevenster \& Veldkamp (1983).

Para el estudio de la flora europea y mediterránea, considero no sólo posible, sino conveniente, mantener un concepto estrecho de los géneros de Gramíneas, en base a tres argumentos principales:

a) Aquellos géneros que cuentan con un número suficiente de especies, como es el caso de Avenula y de Helictotrichon (sensu stricto), presentan de por sí cierta heterogeneidad taxonómica que llevaría a la necesidad de establecer una engorrosa clasificación infragenérica en varias categorías (véase Gervais, 1973, Romero Zarco, 1984a, 1984b, y Röser, 1989). Esta clasificación no puede verse reflejada en la nomenclatura binomial, con la consiguiente pérdida de información conceptual en el caso de la fusión de géneros.

b) La separación de Avenula y Helictotrichon, en lo que respecta a las especies árticas, no sólo se fundamenta en los criterios morfológicos y anatómicos descritos por Holub (1962) y Gervais (1968), sino que también existen ciertas pruebas cariológicas y reproductivas en especies mediterráneas. El cariograma de los diploides y tetraploides del género Avenula presenta una asimetría intracromosómica ligeramente superior a la de los mismos niveles de ploidía del género Helictotrichon (Romero Zarco, 1986). Más interesante aún es el resultado de los cruces interespecíficos e intergenéricos realizados por Gervais (1983), quien concluye que en base a los ensayos de interfertilidad, el género Avena está más estrechamente relacionado con Avenula que con Helictotrichon, y hasta la fecha no se ha obtenido ningún resultado en los cruces Avenula x Helictotrichon y sí se han obtenido híbridos viables, e incluso fértiles dentro de cada género.

c) La existencia de especies intermedias entre los géneros de Gramíneas, es un hecho incuestionable (véanse los abundantes comentarios al respecto en Clayton \& Renvoize, 1986). En el caso que nos ocupa, si se esgrimen ciertas especies como argumento para incluir Avenula en Helictotrichon, también deben tenerse en cuenta otras para unir éste con Arrhenatherum, Pseudarrhenatherum, Avena, e incluso Trisetum (véase Baum, 1968 o Sevenster \& Veldkamp, 1983 respecto al último género). Esto nos llevaría a volver a un concepto linneano revisado del género Avena, lo cual ciertamente es una opción válida, pero poco recomendable en base al argumento expuesto en primer lugar.

Dos cuestiones nomenclaturales afectan a los táxones andaluces relacionados con Avenula marginata (Lowe) J. Holub. De una parte, la prioridad del epíteto específico marginata Love (1938) sobre sulcata Gay ex Boissier (1838) ha sido defendida por Röser (1989), criterio que debe respetarse en ausencia de nuevos datos. En segundo lugar, uno de los táxones del grupo, originalmente descrito con el nombre Avenochloa albinervis subsp. occidentalis Gervais, ha sido recientemente redescrito por Röser (1992) como Helictotrichon cintranum, en base a la invali- 
dez del primer nombre que al parecer carece de tipo adecuado.

\section{Tratamiento taxonómico propuesto.}

\section{Avenula marginata (Love) J. Holub, Preslia} (Praha) 49:219 (1977) subsp. marginata var. marginata

Basiónimo: Avena marginata Love Trans. Cambr. Philos. Soc. 6: 529 (prius V, 1838). Tipo: Madeira. «800. Avena marginata, nob., Rocks at the Boca do Bouraco above the Icehause, Aug. 14.1837» (BM lectotipo, Röser 1989: 136).

= Avena sulcata Gay ex Boiss., Elenchus 88 (V, 1838). Tipo: Asturias. Hab. in pascuis alpinis locus ditisim. Pico de Arvás, 13.VIII, Durieu Pl. Select. Hispano Lusit. sect. $1^{\text {a }}$ Asturicae, Anno 1835, colectae no 176 (G, lectotipo!, Kerguélen, 1978: 394).

= Avenula sulcata (Gay ex Boiss.) Dumort., Bull. Soc. Bot. Belg. 7: 68 (1868).

= Helictotrichon sulcatum (Gay ex Boiss.) Henrard, Blumea 3: 430 (1940).

Avenula marginata subsp. sulcata (Gay ex Boiss.) Franco, Bot. J. Linn. Soc. 78: 236 (1979).

= Helictotrichon marginatum (Lowe) Röser, Dissert. Bot. 145: 136 (1989)

Distribución: Oeste y Sur de Francia, Norte, centro y Oeste de la Península Ibérica, Madeira y Norte de Marruecos. Hasta la fecha no se han encontrado en Andalucía plantas idénticas al tipo de la especie, no obstante, Röser (1989: 137) incluye en este taxon ciertas plantas de la provincia de Cádiz que aquí se adscriben a la subespecie gaditana. Las localidades más próximas son del Algarve (Sierra de Monchique, ELVAS 27446) y de Badajoz (Segura de León, SEV 79507).

\section{Avenula marginata subsp. marginata var.} reuteri (Romero Zarco) Romero Zarco comb. nov.

Basiónimo: Avenula sulcata subsp. sulcata var. reuteri Romero Zarco, Lagascalia 13: 122 (1984). Tipo: Sierra de Guadarrama ad viam Navacerrada... 16 Jul. 1848 (scripsit Reuter, G, holotipo!).

= Avenula marginata subsp. pyrenaica $\mathrm{J}$.
Holub, Preslia 49: 208 (1977). Tipo: Dr. C. Baenitz Herbarium Europaeum, Avena sulcata Gay; Gédre (Hautes Pyrénées); Tertre du Puron, 1050 m, 7.84; leg. Bordére (PR, holotipo). Isoparatipo: Gédre, Ht. Pyr. aout 1864, Bordére (G!).

= Avenula delicatula Franco, Bot. J. Linn. Soc. 76: 359 (1978). Tipo: Miranda do Dauro: Cicomo, Monte de $\mathrm{N}^{\mathrm{a}}$ Sra. da Luz, $900 \mathrm{~m}$., 26.VI.1972, Marcos \& Almeida (LISI). Paratipo: Bragança, Cab. de S. Bartolomeu, V.1884, A. Moller (LISU P-55779!).

La separación de esta variedad de pequeño porte, adaptada a los suelos pobres de arenas graníticas o de rocas ultrabásicas, tiene interés desde el punto de vista ecológico y nomenclatural, ya que ha recibido tratamiento específico o subespecífico en obras florísticas importantes.

Distribución: Pirineos y sistemas montañosos que rodean a la Meseta Norte de la Península Ibérica (Romero Zarco, 1984a: 123).

\section{Avenula marginata subsp. gaditana (Ro-} mero Zarco) Romero Zarco, comb. nova Basiónimo: Avenula sulcata subsp. gaditana Romero Zarco, Lagascalia 13: 124 (1984). Tipo: Cádiz, Conil, Cabo Roche, pinares, 1.V.1981, Romero (SEV 79488, holotipo!)

Distribución y variabilidad: Sur y Oeste de la Península Ibérica. En Andalucía se encuentra en la provincia de Cádiz asociada a matorral sobre suelos arenosos litorales o sobre areniscas (Romero Zarco, 1.c.: 124). En otras zonas del occidente peninsular se encuentran plantas semejantes al tipo gaditano junto a otras intermedias respecto al tipo de la especie.

\section{Avenula marginata subsp. cintrana (Röser)}

Romero Zarco, comb. nova

Basiónimo: Helictotrichon cintranum Röser, Taxon 41: 60 (1992). Tipo: Estremadura, zona Sur de Serra da Sintra, entre Estoril y Sintra, cercanías de la colonia Ribeira da Penha Longa... 1.VII.1985, Röser Av 174 (TUB, holotipo).

= Avenochloa albinervis subsp. occidentalis Gervais, Dekschr. Schweiz. Naturf. Ges. 88: 122 (1973), nomen invalidum sine typo sicco (Röser, 1992). 
= Avenula sulcata subsp. occidentalis Romero Zarco, Lagascalia 13: 124 (1984), nomen invalidum.

$=$ Helictotrichon occidentale Röser, Diss. Bot . 145: 154 (1989) nomen invalidum.

Distribución y variabilidad: La distribución de este taxon se adapta aproximadamente a la provincia corológica Luso-Extremadurense (Rivas Martínez, 1973), encontrandose, en lo que respecta a Andalucía, en enclaves aislados de Sierra Morena (Huelva, Córdoba y Jaén, Romero Zarco, 1.c.: 126) y en dos localidades de las provincias de Cádiz y Málaga (Aparicio \& Silvestre, 1987: 196) sobre afloramientos de areniscas.

En el centro y SW de Portugal se encuentran poblaciones bien caracterizadas desde el punto de vista morfológico y anatómico, sin embargo por toda su área de distribución existen plantas de afinidad dudosa entre esta subespecie y las restantes. El tipo designado por Röser (1992) figura en la relación de hexaploides $(2 n=42)$ del estudio cariológico del mismo autor (Röser, 1989:20). Este citotipo hexaploide sólo ha sido encontrado hasta la fecha en la zona litoral del centro y Sur de Portugal, en la que también se encuentran plantas tetraploides.

\section{Avenula marginata subsp, albinervis}

(Boiss.) Romero Zarco, comb. nova

Basiónimo: Avena albinervis Boiss., Voy. Bot. Midi Esp. 2: 656 (1844). Tipo: Sa. d'Estepona, en haut, Juin (scripsit Boissier, G lectotipo!, Charpin \& Fernández Casas in Laínz 1974: 23).

$=$ Helictotrichon albinerve (Boiss.) Henrard, Blumea 3: 429 (1940).

= Avenula albinervis (Boiss.) Laínz, Comm. Inst. Nac. Invest. Agrar. Ser. Recurs. Nat. (Madrid) 2: 23 (1974).

= Avenula sulcata subsp. albinervis (Boiss.) Rivas Martínez, Acta Bot. Malacitana 2: 63 (1976).

Distribución y variabilidad. Oeste y Sur de la Península Ibérica, Norte de Marruecos. En el territorio andaluz es relativamente abundante respecto al resto de los táxones de la especie, encontrandose en pastizales de talu- des y claros del matorral sobre suelos ácidos o peridotíticos, en las sierras de las provincias de Cádiz y Málaga (Romero Zarco, 1.c.: 127).

El citotipo diploide, correspondiente a la localidad clásica y otras próximas de la provincia de Cádiz (Röser, 1989: 20; Romero Zarco, 1.c.: 45), es claramente diferenciable de la subespecie cintrana, sin embargo entre los tetraploides que se distribuyen por todo el área del taxon, es posible encontrar plantas intermedias entre ambas subespecies. Este patrón de variabilidad es frecuente en otros complejos diploides-tetraploides, como el caso de Avena barbata (Romero Zarco, 1990) o Dactylis glomerata (Borril, 1961: 450), por citar dos casos conocidos del occidente de la Región Mediterránea.

\section{BIBLIOGRAFÍA}

APARICIO, A.y S.SILVESTRE - 1987- Flora del Parque Natural de la Sierra de Grazalema. Junta de Andalucía, Agencia de Medio Ambiente, Sevilla.

BAUM, B.R. -1968- Delimitation of the genus Avena (Gramineae). Can. Jour. Bot., 46: 121132.

BOR, N.L. -1968- Gramineae, in C.C. Townsend, E. Guest \& A. Al-Rawi (eds.), Flora of Iraq 9. Ministry of Agriculture of the Republic of Iraq, Bhagdad.

BORRILL, M. -1961- Dactylis marina. A natural group of related tetraploid forms. J. Lin. Soc (Bot.), 56(368): 431-439

CLAYTON, W.D. \& S.A. RENVOIZE -1986Genera Graminum. Grasses of the world. Kew Bulletin Additional Series XIII. Royal Botanic Gardens, Kew. Her Majesty's Stationary Office, London

DUVAL-JOUVE, J.M. -1863- Notes sur les caractéres que les arétes et les feuilles peuvent fournir pour la division en sections du genre Avena. Bull. Soc. Bot. France, 10: 50-55.

GERVAIS, C. -1968- Sur une critère anatomique nouveae, utilizable dans la taxinomie des avoines vivaces. Bull. Soc. Bot. Suisse, 78: 369-372 
GERVAIS, C. -1973- Contribution à l'étude cytologique et taxonomique des avoines vivaces. Denksch. Schweiz. Naturf. Ges., 88: 3-166.

GERVAIS, C. -1983- Wide hybridization attemps in the tribe Aveneae Nees. Bot. Helv., 93:195212

HENRARD, J. TH. -1940- Notes on the nomenclature of some grasses. Blumea, 3: 411480.

HITCHCOCK, A.S. -1950- Manual of the Grasses of the United States, ed. 2, U.S. Goverment Print Off., Misc. Publication No. 200, Washington D. C.

HOLUB, J. -1958- Bemerkungen zur Taxonomie der Gattum Helictotrichon Bess., in KLASTERSKY, I. et al. (EDS) Philips Maxmilian Opiz und seine Badentung für die Pflanzentaxonomie: 101-133. Pragha.

HOLUB, J. - 1962- Ein Beitrag zur Abgrenzung der Gattungen in der Tribus Aveneae: die Gattung Avenochloa Holub. Acta Horti Bot. Prag., 1962: 75-86.

KERGUÉLEN, M. -1978- Notes agrostologiques. IV. Bull. Soc. Bot. France, 125(7-8): 391-400.

LAÍNZ, M. -1974- Aportaciones al conocimiento de la flora gallega, VIII. Comun. Inst. Nac. Invest. Agrar. Ser. Recurs. Nat. (Madrid), 1974(2): 1-26.

PAUNERO, E. - 1959a- Las Aveneas españolas IV. Anales Jard. Bot. Madrid, 17(1): 257-376.

POTZTAL, E. -1951- Anatomisch-systematische Untersuchungen an den Gattungen Arrhenatherum und Helictotrichon. Bot. Jahrb., 75: $321-332$

RIVAS MARTÍNEZ, S. - 1973- Avances sobre una síntesis corológica de la Península Ibérica, Baleares y Canarias. Anal. Inst. Bot. Cavanilles, 30: 69-87.

ROMERO ZARCO, C. -1984a- Revisión taxonómica del género Avenula (Dumort.)
Dumert. (Gramineae) en la Península Ibérica e Islas Baleares. Lagascalia, 13: 39-146.

ROMEROZARCO, C. -1984b- Revisión del género Helictotrichon Besser ex Schultes \& Schultes fil. (Gramineae) en la Península Ibérica. I. Estudio taxonómico. Anales Jard. Bot. Madrid, 41: 97-124.

ROMERO ZARCO, C. -1986- A new method for estimating karyotype asymmetry. Taxon, 35: 526-530.

ROMERO ZARCO, C. -1990- Las avenas del grupo barbata en la Península Ibérica y Baleares. Lagascalia, 16(2): 243-268.

RÖSER, M. -1989- Karyologische, systematische und chorologische Untersuchungen an der Gattung Helictotrichon Besser ex Schultes \& Schultes (Poaceae) im westlichen Mittelmeergebiet. Dissert. Bot., 145: 1-250.

RÖSER, M. -1992- Helictotrichon cintranum, species nova, a rare south-west European oat grass (Poaceae: Pooideae: Aveneae). Taxon, 41: 60-61.

SEVENSTER, J.G. \& J.F. VELDKAMP - 1983- A revision of Helictotrichon (Gramineae) in Malesia. Blumea, 28: 329 -342

WATSON, L. \& M.J. DALLWITZ -1992- The grass genera of the world. C.A.B. International, Cambridge.

Aceptado para su publicación en Junio de 1993

Dirección del autor. Departamento de Biología Vegetal y Ecología, Facultad de Biología, Universidad de Sevilla, Apartado 1095, 41080Sevilla, España. 